\title{
Synthesis and Structure Activity Relationship of Some Indole Derivatives as Potential Anti-inflammatory Agents
}

\author{
Samar S. Fatahala, ${ }^{1 *}$ Mohammed A. Khedr ${ }^{2}$ \\ and Mossad S. Mohamed ${ }^{1}$ \\ ${ }^{1}$ Pharmaceutical Organic Chemistry Department, Faculty of Pharmacy, Helwan University, Ain-Helwan \\ ${ }^{2}$ Pharmaceutical Chemistry Department, Faculty of Pharmacy, Helwan University, Ain-Helwan \\ Postal code: 11795, Helwan, Cairo, Egypt \\ *Corresponding author: E-mail: ssfathatllah@yahoo.com, samarradwan1@yahoo.com
}

Received: $25-04-2017$

\begin{abstract}
A series of fused pyrroles were synthesized and tested for their in vivo anti-inflammatory activity. Among 14 examined derivatives, 5 derivatives ( $\mathbf{1} \mathbf{b}-\mathbf{e}, \mathbf{g}$ and $\mathbf{5 b}$ ), showed a promising anti-inflammatory activity equivalent to reference anti-inflammatory drugs (indomethacin and ibuprofen). A molecular docking study was conducted to interpret the biological activities of the tested compounds. The docking results were complementary with the phase of the biological survey and confirmed the biological effects.
\end{abstract}

Keywords: Fused pyrroles, tetrahydroindoles, Molecular Docking, anti-inflammatory assay

\section{Introduction}

Non-steroid anti-inflammatory drugs (NSAIDs) have been amongst the most widely developed drugs. ${ }^{1-5}$ They have provided an alternative to steroid therapy, which has revealed many problems related to parallel endocrine and metabolic activity, induced osteoporosis and hypercalcemia, as shown by Lessigiarska et al. ${ }^{\text {Th }}$ The postponement in treatment causes severe side effects including rhinnorrhoea, rheumatoid arthritis, and atherosclerosis. ${ }^{7}$ With their anti-pyretic and analgesic activities, they represent a choice treatment in various inflammatory diseases such as arthritis and rheumatisms. ${ }^{8-10} 5$-dimethyl-3-oxo-2phenyl-2,3-dihydro-1H-pyrazol-4-yl The NSAIDs have exerted their anti-inflammatory activities through cyclooxygenase (COX) inhibition. ${ }^{11-13}$

Structural variation of the heterocyclic rings through the manipulation of the heterocyclic core influences the activity of the resulting fused systemes, among these of pyrroles and their fused deivatives. ${ }^{14-16}$ Due to their pharmaceutical importance, ${ }^{17-22}$ attention was paid to develop a new synthetic route for pyrroles and their fused forms. ${ }^{23-28}$ Pyrrolylacetic acid derivatives such as tolmetin $\left(\right.$ Rumatol $\left.^{\circledR}\right)$ and zomepirac $\left(\right.$ Zomax $\left.^{\circledR}\right)$ were proved to be NSAIDs ${ }^{6}$ with strong anti-inflammatory activity. ${ }^{29,30}$ Other pyrrole and fused pyrrole compounds have been recently reported as potent COX-1 and COX2 inhibitors: ${ }^{31,32} 4$-benzodioxine or pyrrole nucleus are described. All the newly synthesized compounds were examined for their in vitro and in vivo anti-inflammatory activity. Several derivatives, including ( $S$ indomethacin $\left(\right.$ Indacin ${ }^{\circledR}$ ), acemetacin $\left(\right.$ Emflex $\left.^{\circledR}\right)$ and etodolac (Eto$\left.\operatorname{din}^{\circledR}\right)$ as indole derivatives, and ketorolac $\left(\operatorname{Ketolac}^{\circledR}\right)$ as a pyrrole derivative. ${ }^{33-36}$ These compounds blocked prostaglandin synthesis by non-selective inhibition of COX-1 and COX-2 (indomethacin, acemetacin, tolmetin and ketorolac) or by selective inhibition of COX-2 (etodolac) (Fig. 1).

Due to the importance of this ring system, ${ }^{39}$ we prepared some fused $o$-aminocyano tetrahydrobenzo[b]pyrrole derivatives as an essential propagation step in our search for new pyrrole and pyrrolopyrimidine derivatives, ${ }^{40-43}$ and evaluated them for the anti-inflammatory activities. In addition, a molecular docking study has been done to explain the activity of the biologically active compounds. 

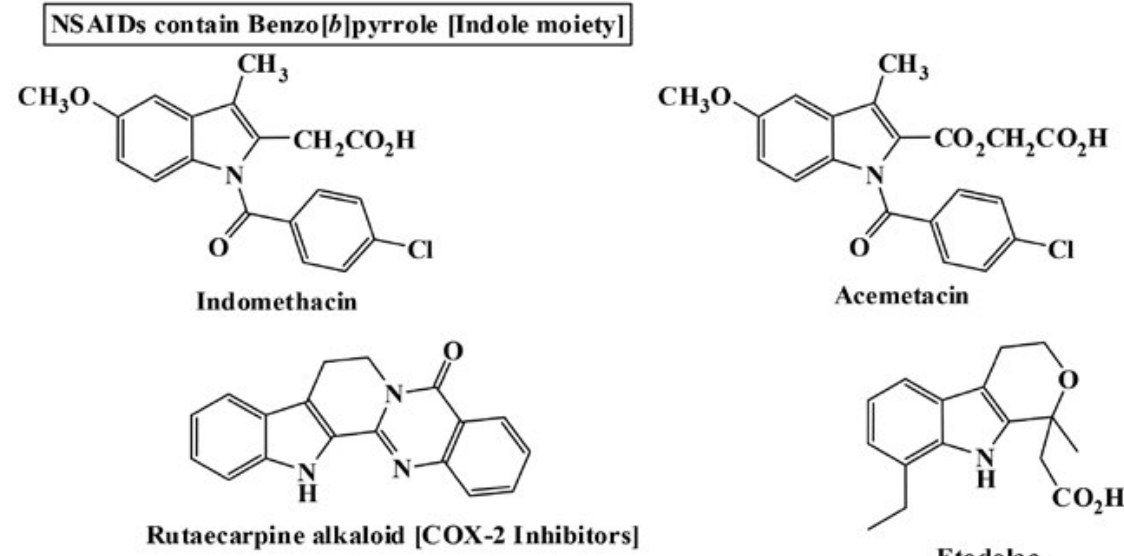

Rutaecarpine alkaloid [COX-2 Inhibitors]

NSAIDs contain Pyrrole moiety

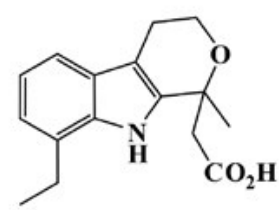

Etodolac
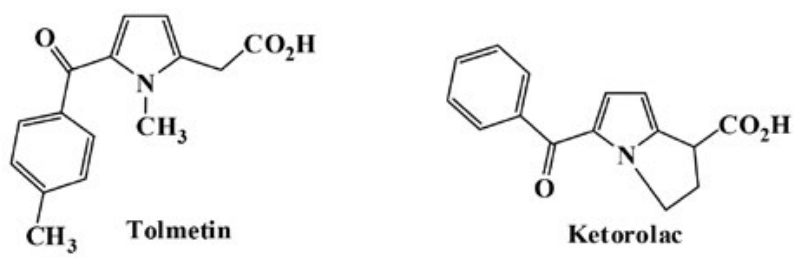

Figure 1: Pyrroles and Indoles derivatives as NSAIDs ${ }^{37,38}$ their mechanism of action at the molecular level such as cyclooxygenase (COX

\section{Experimental}

\section{1. Chemistry}

\section{General Information for Chemicals}

All melting points were uncorrected and measured using Electro-thermal IA 9100 apparatus (Shimadzu, Japan). IR spectra were recorded as potassium bromide pellets on a Perkin-Elmer 1650 spectrophotometer (USA), Faculty of Science, Cairo University, Cairo, Egypt. ${ }^{1} \mathrm{H}$-NMR and ${ }^{13} \mathrm{C}$-NMR spectra were performed on Jeol NMR FXQ-300 $\mathrm{MHz}$ and Jeol NMR FXQ-500 $\mathrm{MHz}$ spectrometers; chemical shifts are expressed as ppm against TMS as the internal reference (Faculty of Science, Cairo University, Cairo, Egypt). Mass spectra were recorded at $70 \mathrm{eV}$ EI Ms-QP 1000 EX (Shimadzu, Japan), Faculty of Science, Cairo University, Cairo, Egypt. Microanalyses were operated using Vario, Elemental apparatus (Shimadzu, Japan), Organic Microanalysis Unit, Faculty of Science, Cairo University, Cairo, Egypt. Column Chromatography was performed on (Merck) Silica gel 60 (particle size 0.06$0.20 \mathrm{~mm}$ ). Compounds $\mathbf{1 a}, \mathbf{b}, \mathbf{3 a}, \mathbf{b}$ and $\mathbf{5 a}$ were prepared as reported in the literature. ${ }^{44}$ All new compounds yielded spectral data consistent with the proposed structures and microanalysis within $\pm 0.4 \%$ of the theoretical values.

General methods for the preparation of 2-oxo-substituded malononitriles $\mathbf{I}$ and $\mathbf{I I}$

A mixture of 2-chloroketone $(0.01 \mathrm{~mol})$ and malononitrile $(1 \mathrm{~g}, 0.016 \mathrm{~mol})$ in $\mathrm{CHCl}_{3}(50 \mathrm{~mL})$, was cooled with stirring to $0-5{ }^{\circ} \mathrm{C}$ for $30 \mathrm{~min}$. A cold solution of
$\mathrm{NaOH}(2.5 \mathrm{~g}$ in $10 \mathrm{~mL}$ of water) was added to the mixture dropwise for $30 \mathrm{~min}$. The stirring was continued for 50 min under ice and the reaction mixture then left for $72 \mathrm{~h}$ at room temperature. The precipitate formed was filtered off, recrystallized from appropriate solvent.

\section{2-(2-Oxocyclohexyl)malononitrile (I)}

It was obtained as a brownish red solid $\left[\mathrm{EtOH} / \mathrm{H}_{2} \mathrm{O}\right]$. Yield 64\%, m.p. $142-145^{\circ} \mathrm{C}$, FT-IR (KBr) $v_{\max } 2345,1690$ $\mathrm{cm}^{-1}$, EIMS $\mathrm{m} / z(\%) 162\left[\mathrm{M}^{+}\right]$(7.5), 106 (100). Anal. Calcd for $\mathrm{C}_{9} \mathrm{H}_{10} \mathrm{~N}_{2} \mathrm{O}: \mathrm{C}, 66.65 ; \mathrm{H}, 6.21 ; \mathrm{N}, 17.27$. Found: $\mathrm{C}, 66.98$; $\mathrm{H}, 6.54 ; \mathrm{N}, 17.03$.

\section{2-(1-Oxo-2,3-dihydro-1H-inden-2-yl)malononitrile (II)}

It was obtained as a reddish brown solid [EtOH/ $\mathrm{H}_{2} \mathrm{O}$ ]. Yield $65 \%$, m.p. $142-147^{\circ} \mathrm{C}$, FT-IR (KBr) $v_{\max } 2360$, $1705 \mathrm{~cm}^{-1}$, EIMS m/z (\%) $196\left[\mathrm{M}^{+}\right]$(4), 144 (100). Anal. Calcd for $\mathrm{C}_{12} \mathrm{H}_{8} \mathrm{~N}_{2} \mathrm{O}: \mathrm{C}, 73.46 ; \mathrm{H}, 4.11 ; \mathrm{N}, 14.28$. Found: C, $73.78 ; \mathrm{H}, 4.43 ; \mathrm{N}, 14.60$.

\section{2-Amino-1-(aryl)-4,5,6,7-tetrahydro-1H-indole-3-carboni-} trile $\mathbf{1 a - g}$

Method A: A solution of I ( $1 \mathrm{~g}, 0.016 \mathrm{~mol})$ in ethanol $(30 \mathrm{~mL})$, the appropriate aromatic amine $(0.01 \mathrm{~mol})$ and conc $\mathrm{HCl}(4 \mathrm{~mL})$ were added. The reaction mixture was refluxed for $72 \mathrm{~h}$ (TLC monitored), cooled to room temperature, poured onto crushed ice (25 g.), and neutralized with $\mathrm{NaOH}$. The precipitate formed was filtered off, dried and recrystallized from appropriate solvent to give compound 1. 
Method B: A solution of $\mathbf{I}(1 \mathrm{~g}, 0.016 \mathrm{~mol})$ in isopropanol $(30 \mathrm{~mL})$, the appropriate aromatic amine $(0.01 \mathrm{~mol})$ was added. The reaction mixture was refluxed for $72 \mathrm{~h}$ (TLC monitored), then cooled to room temperature, and the solvent was removed under reduce pressure. The residue was dissolved in methanol $(10 \mathrm{~mL})$, poured into crushed ice $(25 \mathrm{~g})$. The precipitate formed was filtered off, dried and recrystallized from appropriate solvent to give compound 1

\section{2-Amino-1-(4-chlorophenyl)-4,5,6,7-tetrahydro-1H-indole- 3-carbonitrile (1c)}

It was obtained as a brown solid [EtOH]. Yield for method A) $73 \%$ and for B) $52 \%$, m.p. $184-188^{\circ} \mathrm{C}$. FT-IR (KBr) $v_{\max } 3380-3250,2315 \mathrm{~cm}^{-1} .{ }^{1} \mathrm{H}-\mathrm{NMR}$ (DMSO- $d_{6}$, $300 \mathrm{MHz}) \delta=1.63-1.88\left(\mathrm{~m}, 4 \mathrm{H}, 2 \times \mathrm{CH}_{2}\right), 2.5-2.78(\mathrm{~m}$, $4 \mathrm{H}, 2 \times \mathrm{CH}_{2}$ ), 5.78 (br.s, $2 \mathrm{H}, \mathrm{NH}_{2}, \mathrm{D}_{2} \mathrm{O}$ exchangeable), 7.2-7.8 (m, $4 \mathrm{H}, \mathrm{Ar}-\mathrm{H}) .{ }^{13} \mathrm{C}$ NMR (DMSO- $\left.d_{6}\right): \delta 34.1\left(\mathrm{CH}_{2}\right.$, $\mathrm{C}-4), 34.9\left(\mathrm{CH}_{2}, \mathrm{C}-7\right), 35.1\left(\mathrm{CH}_{2}, \mathrm{C}-5\right), 36.2\left(\mathrm{CH}_{2}, \mathrm{C}-6\right)$, $116.1\left(\mathrm{~N}-{ }^{*} \mathrm{C}=\mathrm{C}, \mathrm{C}-4 \mathrm{a}\right), 116.9\left(\mathrm{~N}-\mathrm{C}=\mathrm{C}^{*}, \mathrm{C}-7 \mathrm{a}\right), 119.8$ $\left(\mathrm{C}^{\prime} \mathrm{N}\right), 124.1$ (C-2), $125.6(\mathrm{C}-3), 130.9\left(\mathrm{CH}\right.$ aromatic, $\left.\mathrm{C}-2^{\prime}\right)$, $132.2\left(\mathrm{CH}\right.$ aromatic, $\left.\mathrm{C}-3^{\prime}\right), 132.8\left(\mathrm{CH}\right.$ aromatic, $\left.\mathrm{C}-5^{\prime}\right)$, 133.4 (CH aromatic, C-6'), 134.1(C-Cl, C-4'), $145.2(\mathrm{C}-\mathrm{N}$, C-1') ppm; EIMS $m / z(\%) 271\left[\mathrm{M}^{+}\right](25), 273\left[\mathrm{M}^{+}+2,{ }^{37} \mathrm{Cl}\right]$ (8.5), 149 (100). Anal. Calcd for $\mathrm{C}_{15} \mathrm{H}_{14} \mathrm{ClN}_{3} ; \mathrm{C}, 66.30 ; \mathrm{H}$, 5.19; N, 15.46. Found: C, 66.68; H, 5.56; N, 15.30 .

2-Amino-1-o-tolyl-4,5,6,7-tetrahydro-1H-indole-3-carbonitrile (1d)

It was obtained as a brownish red solid $\left[\mathrm{EtOH} / \mathrm{H}_{2} \mathrm{O}\right]$. Yield for method A) $80 \%$, B) $52 \%$, m.p. $195-197^{\circ} \mathrm{C}$. FT-IR (KBr) $v_{\max } 3380-3280,2285 \mathrm{~cm}^{-1} .{ }^{1} \mathrm{H}-\mathrm{NMR}$ (DMSO- $d_{6}$, $300 \mathrm{MHz}) \delta=1.53-1.78\left(\mathrm{~m}, 4 \mathrm{H}, 2 \times \mathrm{CH}_{2}\right), 2.12(\mathrm{~s}, 3 \mathrm{H}$, $\left.\mathrm{CH}_{3}\right), 2.5-2.78\left(\mathrm{~m}, 4 \mathrm{H}, 2 \times \mathrm{CH}_{2}\right), 6.18$ (br.s, $2 \mathrm{H}, \mathrm{NH}_{2}, \mathrm{D}_{2} \mathrm{O}$ exchangeable), 7.2-7.6 (m, 4H, Ar-H) ppm. EIMS m/z (\%) $251\left[\mathrm{M}^{+}\right](31), 149$ (100). Anal. Calcd for $\mathrm{C}_{16} \mathrm{H}_{17} \mathrm{~N}_{3}: \mathrm{C}$, 76.46; H, 6.82; N, 16.72. Found: C, 76.86; H, 6.41; N, 16.77.

\section{2-Amino-1-p-tolyl-4,5,6,7-tetrahydro-1H-indole-3-carbo-} nitrile (1e)

It was obtained as a brown solid $\left[\mathrm{EtOH} / \mathrm{H}_{2} \mathrm{O}\right]$. Yield for method A) $80 \%$, B) $54 \%$, m.p. $168-172^{\circ} \mathrm{C}$. FT-IR (KBr) $v_{\max } 3390-3270,2260 \mathrm{~cm}^{-1} .{ }^{1} \mathrm{H}-\mathrm{NMR}$ (DMSO- $d_{6}, 300$ $\mathrm{MHz}) \delta=1.53-1.78\left(\mathrm{~m}, 4 \mathrm{H}, 2 \times \mathrm{CH}_{2}\right), 1.98\left(\mathrm{~s}, 3 \mathrm{H}, \mathrm{CH}_{3}\right)$, 2.4-2.78 (m, $\left.4 \mathrm{H}, 2 \times \mathrm{CH}_{2}\right), 5.58$ (br.s, $2 \mathrm{H}, \mathrm{NH}_{2}, \mathrm{D}_{2} \mathrm{O} \mathrm{ex}-$ changeable), 7.2-7.8 (m, $4 \mathrm{H}$, Ar-H) ppm. EIMS $\mathrm{m} / z(\%)$ $251\left[\mathrm{M}^{+}\right](61), 92(100)$. Anal. Calcd for $\mathrm{C}_{16} \mathrm{H}_{17} \mathrm{~N}_{3}: \mathrm{C}, 76.46$; H, 6.82; N, 16.72. Found: C, 76.43; H, 6.91; N, 16.97.

2-Amino-1-(1,5-dimethyl-3-oxo-2-phenyl-2,3-dihydro- $1 \mathrm{H}$ -pyrazol-4-yl)-4,5,6,7-tetrahydro-1H-indole-3-carbonitrile (1f)

It was obtained as a yellowish brown solid [EtOH/ $\mathrm{H}_{2} \mathrm{O}$ ]. Yield for method A) $76 \%$, B) $55 \%$, m.p. $234-238^{\circ} \mathrm{C}$. FT-IR (KBr) $v_{\max } 3350-3270,2270,1680 \mathrm{~cm}^{-1} .{ }^{1} \mathrm{H}-\mathrm{NMR}$ (DMSO- $\left.d_{6}, 300 \mathrm{MHz}\right) \delta=1.43-1.68\left(\mathrm{~m}, 4 \mathrm{H}, 2 \times \mathrm{CH}_{2}\right)$,
2.2-2.45 (m, $\left.4 \mathrm{H}, 2 \times \mathrm{CH}_{2}\right), 2.33\left(\mathrm{~s}, 3 \mathrm{H}, \mathrm{CH}_{3}\right), 3.12(\mathrm{~s}, 3 \mathrm{H}$, $\mathrm{N}-\mathrm{CH}_{3}$ ), 5.28 (br.s, $2 \mathrm{H}, \mathrm{NH}_{2}, \mathrm{D}_{2} \mathrm{O}$ exchangeable), 7.2-7.6 (m, 5H, Ar-H) ppm. EIMS m/z (\%) $347\left[\mathrm{M}^{+}\right]$(38), 173 (100). Anal. Calcd for $\mathrm{C}_{20} \mathrm{H}_{21} \mathrm{~N}_{5} \mathrm{O}: \mathrm{C}, 69.14 ; \mathrm{H}, 6.09 ; \mathrm{N}$, 20.16; O, 4.61. Found: C, 69.28; H, 6.16; N, 20.30; O, 4.30.

2-Amino-1-(pyridin-2-yl)-4,5,6,7-tetrahydro-1H-indole-3carbonitrile (1g)

It was obtained as a brownish black solid [EtOH]. Yield for method A) $65 \%$, B) $48 \%$, m.p. $194-196{ }^{\circ} \mathrm{C}$. FT-IR (KBr) $v_{\max } 3370-3290,2345 \mathrm{~cm}^{-1} .{ }^{1} \mathrm{H}-\mathrm{NMR}$ (DMSO- $d_{6}$, $300 \mathrm{MHz}) \delta=1.7-1.75\left(\mathrm{~m}, 4 \mathrm{H}, 2 \times \mathrm{CH}_{2}\right), 2.6-2.8(\mathrm{~m}, 4 \mathrm{H}, 2$ $\times \mathrm{CH}_{2}$ ), 5.28 (br.s, $2 \mathrm{H}, \mathrm{NH}_{2}, \mathrm{D}_{2} \mathrm{O}$ exchangeable), 7.6-8.4 (m, 4H, Ar-H) ppm. EIMS m/z (\%) 238 [M $\mathrm{M}^{+}$(100). Anal. Calcd for $\mathrm{C}_{14} \mathrm{H}_{14} \mathrm{~N}_{4}$ : C, 70.57; H, 5.92; N, 23.51. Found: $\mathrm{C}, 70.71 ; \mathrm{H}, 5.83 ; \mathrm{N}, 23.46$.

\section{2-Amino-1-(aryl)-4,5,6,7-tetrahydro-1H-indole-3-carboni-} trile $2 a, b$

A solution of II ( $1 \mathrm{~g}, 0.016 \mathrm{~mol})$ in ethanol $(30 \mathrm{~mL})$, the appropriate aromatic amine $(0.01 \mathrm{~mol})$ and conc $\mathrm{HCl}$ $(4 \mathrm{~mL})$ were added. The reaction mixture was refluxed for $72 \mathrm{~h}$ (TLC monitored), then cooled to room temperature, poured into crushed ice $(25 \mathrm{~g})$, and neutralized with $\mathrm{NaOH}$. The precipitate formed was filtered off, dried and recrystallized from appropriate solvent to give compound 2a,b

2-Amino-1-phenyl-1,4-dihydroindeno[1,2-b]pyrrole-3-carbonitrile (2a)

It was obtained as a brownish black solid [EtOH/ $\mathrm{H}_{2} \mathrm{O}$. Yield 60\%, m.p. $218-220^{\circ} \mathrm{C}$. FT-IR (KBr) $v_{\max } 3410-$ $3240,2335 \mathrm{~cm}^{-1} .{ }^{1} \mathrm{H}-\mathrm{NMR}$ (DMSO- $\left.d_{6}, 300 \mathrm{MHz}\right) \delta=3.4(\mathrm{~s}$, $2 \mathrm{H}, \mathrm{CH}_{2}$ ), 6.18 (br.s, $2 \mathrm{H}, \mathrm{NH}_{2}, \mathrm{D}_{2} \mathrm{O}$ exchangeable), 6.8-7.4 (m, 9H, Ar-H) ppm. EIMS m/z (\%) $271\left[\mathrm{M}^{+}\right]$(100). Anal. Calcd for $\mathrm{C}_{18} \mathrm{H}_{13} \mathrm{~N}_{3} ; \mathrm{C}, 79.68 ; \mathrm{H}, 4.83 ; \mathrm{N}, 15.49$. Found: $\mathrm{C}$, 79.89; H, 4.97; N, 15.80 .

2-Amino-1-(4-methoxyphenyl)-1,4-dihydroindeno[1,2-b] pyrrole-3-carbonitrile (2b)

It was obtained as a brown solid $\left[\mathrm{EtOH} / \mathrm{H}_{2} \mathrm{O}\right]$. Yield 80\%, m.p. $200-205^{\circ} \mathrm{C}$. FT-IR (KBr) $v_{\max } 3425-3220,2340$ $\mathrm{cm}^{-1} .{ }^{1} \mathrm{H}-\mathrm{NMR}$ (DMSO- $\left.d_{6}, 300 \mathrm{MHz}\right) \delta=3.3\left(\mathrm{~s}, 2 \mathrm{H}, \mathrm{CH}_{2}\right)$, $3.7\left(\mathrm{~s}, 3 \mathrm{H}, \mathrm{OCH}_{3}\right), 6.23$ (br.s, $2 \mathrm{H}, \mathrm{NH}_{2}, \mathrm{D}_{2} \mathrm{O}$ exchangeable), 6.8-7.51 (m, 8H, Ar-H) ppm. EIMS $m / z(\%) 301\left[\mathrm{M}^{+}\right]$ (7.6), 211 (100). Anal. Calcd for $\mathrm{C}_{19} \mathrm{H}_{15} \mathrm{~N}_{3} \mathrm{O}: \mathrm{C}, 75.73 ; \mathrm{H}$, 5.02; N, 13.94; O, 5.31. Found: C, 75.61; H, 5.39; N, 14.31; $\mathrm{O}, 5.41$.

\section{$\mathrm{N}$-(3-Cyano-1-(aryl)-pyrrol-2-yl) acetamide 3 and 4}

A suspension of the appropriate compound 1 or 2 $(0.01 \mathrm{~mol})$ in acetic anhydride $(40 \mathrm{~mL})$ was refluxed for 2 $\mathrm{h}$, cooled, poured onto ice-water, neutralized with ammonia to give a precipitate which was filtered off, dried, and recrystallized from appropriate solvent, to give compounds 3 and 4. 
$\mathrm{N}$-(1-(4-Chlorophenyl)-3-cyano-4,5,6,7-tetrahydro-1H-indol-2-yl) acetamide (3c)

It was obtained as a brownish red solid $[\mathrm{MeOH} /$ $\mathrm{H}_{2} \mathrm{O}$ ]. Yield $70 \%$, m.p. $184-188^{\circ} \mathrm{C}$. FT-IR (KBr) $v_{\max } 3300$, $2300,1730 \mathrm{~cm}^{-1} .{ }^{1} \mathrm{H}-\mathrm{NMR}$ (DMSO- $\left.d, 300 \mathrm{MHz}\right) \delta=1.63-$ $1.88\left(\mathrm{~m}, 4 \mathrm{H}, 2 \times \mathrm{CH}_{2}\right), 2.5-2.78\left(7 \mathrm{H},(4 \mathrm{H}) 2 \times \mathrm{CH}_{2}, 3 \mathrm{H}\right.$, $\left.\mathrm{CH}_{3}\right)$ ), 7.2-7.8 (m, 4H, Ar-H), $10.15\left(\mathrm{~s}, 1 \mathrm{H}, \mathrm{NH}, \mathrm{D}_{2} \mathrm{O}\right.$ exchangeable) ppm. EIMS $m / z(\%) 313\left[\mathrm{M}^{+}\right](19.7), 315$ $\left[\mathrm{M}^{+}+2,{ }^{37} \mathrm{Cl}\right]$ (6), 149 (100). Anal. Calcd for $\mathrm{C}_{17} \mathrm{H}_{16} \mathrm{ClN}_{3} \mathrm{O}$ : C, 65.07; H, 5.14; Cl, 11.30; N, 13.39. Found: C, 65.07; H, $5.14 ; \mathrm{Cl}, 11.30 ; \mathrm{N}, 13.39$.

$\mathrm{N}$-(3-Cyano-1-o-tolyl-4,5,6,7-tetrahydro-1H-indol-2-yl) acetamide $(\mathbf{3 d})$

It was obtained as a yellowish brown solid [MtOH/ $\left.\mathrm{H}_{2} \mathrm{O}\right]$. Yield $80 \%$, m.p. $200-205^{\circ} \mathrm{C}$. FT-IR (KBr) $v_{\text {max }} 3280$, $2223,1703 \mathrm{~cm}^{-1} .{ }^{1} \mathrm{H}-\mathrm{NMR}$ (DMSO- $\left.d_{6}, 300 \mathrm{MHz}\right) \delta=1.53-$ $1.78\left(\mathrm{~m}, 4 \mathrm{H}, 2 \times \mathrm{CH}_{2}\right), 2.12\left(\mathrm{~s}, 3 \mathrm{H}, \mathrm{CH}_{3}\right), 2.2-2.58(\mathrm{~m}, 7 \mathrm{H}$, $\left.(4 \mathrm{H}) 2 \times \mathrm{CH}_{2}, 3 \mathrm{H}, \mathrm{CH}_{3}\right), 7.2-7.6(\mathrm{~m}, 4 \mathrm{H}, \mathrm{Ar}-\mathrm{H}), 9.85(\mathrm{~s}$, $1 \mathrm{H}, \mathrm{NH}, \mathrm{D}_{2} \mathrm{O}$ exchangeable) ppm. EIMS $m / z(\%) 293\left[\mathrm{M}^{+}\right]$ (19.7) 92 (100). Anal. Calcd for $\mathrm{C}_{18} \mathrm{H}_{19} \mathrm{~N}_{3} \mathrm{O} ; \mathrm{C}, 73.69 ; \mathrm{H}$, 6.53; N, 14.32. Found: C, 73.99; H, 6.73; N, 14.67.

N-(3-Cyano-1-p-tolyl-4,5,6,7-tetrahydro-1H-indol-2-yl) acetamide (3e)

It was obtained as a reddish black solid $\left[\mathrm{EtOH} / \mathrm{H}_{2} \mathrm{O}\right]$. Yield 70\%, m.p. $186-190^{\circ} \mathrm{C}$. FT-IR (KBr) $v_{\text {max }} 3330,2210$, $1690 \mathrm{~cm}^{-1} .{ }^{1} \mathrm{H}-\mathrm{NMR}$ (DMSO- $\left.d_{6}, 300 \mathrm{MHz}\right) \delta=1.53-1.78$ $\left(\mathrm{m}, 4 \mathrm{H}, 2 \times \mathrm{CH}_{2}\right), 1.98\left(\mathrm{~s}, 3 \mathrm{H}, \mathrm{CH}_{3}\right), 2.2-2.68(\mathrm{~m}, 7 \mathrm{H},(4 \mathrm{H})$ $\left.2 \times \mathrm{CH}_{2}, 3 \mathrm{H}, \mathrm{CH}_{3}\right), 7.2-7.8(\mathrm{~m}, 4 \mathrm{H}, \mathrm{Ar}-\mathrm{H}), 9.5(\mathrm{~s}, 1 \mathrm{H}, \mathrm{NH}$, $\mathrm{D}_{2} \mathrm{O}$ exchangeable) ppm. EIMS $m / z(\%) 293\left[\mathrm{M}^{+}\right](17.7)$, 91 (100). Anal. Calcd for $\mathrm{C}_{18} \mathrm{H}_{19} \mathrm{~N}_{3} \mathrm{O}: \mathrm{C}, 73.69 ; \mathrm{H}, 6.53 ; \mathrm{N}$, 14.32. Found: C, 73.79; H, 6.41; N, 14.39 .

N-(3-Cyano-1-(1,5-dimethyl-3-oxo-2-phenyl-2,3-dihydro1H-pyrazol-4-yl)-4,5,6,7-tetrahydro-1H-indol-2-yl)acetamide (3f)

It was obtained as a brownish red solid $[\mathrm{MeOH} /$ $\mathrm{H}_{2} \mathrm{O}$ ]. m.p. $215-218^{\circ} \mathrm{C}$. FT-IR $(\mathrm{KBr}) v_{\max } 3330,2230,1720$, $1703 \mathrm{~cm}^{-1} .{ }^{1} \mathrm{H}-\mathrm{NMR}$ (DMSO- $\left.d_{6}, 300 \mathrm{MHz}\right) \delta=1.43-1.68$ $\left(\mathrm{m}, 4 \mathrm{H}, 2 \times \mathrm{CH}_{2}\right), 2.2-2.45\left(\mathrm{~m}, 7 \mathrm{H},(4 \mathrm{H}) 2 \times \mathrm{CH}_{2}\right), 2.33-$ $2.4\left(\mathrm{~m}, 6 \mathrm{H}, 2 \times \mathrm{CH}_{3}\right), 3.12\left(\mathrm{~s}, 3 \mathrm{H}, \mathrm{N}-\mathrm{CH}_{3}\right), 7.2-7.6(\mathrm{~m}, 5 \mathrm{H}$, Ar-H). 10.3 (s, 1H, NH, D $\mathrm{O}$ exchangeable) ppm. EIMS $m / z$ (\%) $389\left[\mathrm{M}^{+}\right]$(24.1), 159 (100). Anal. Calcd for $\mathrm{C}_{22} \mathrm{H}_{23} \mathrm{~N}_{5} \mathrm{O}_{2} ; \mathrm{C}, 67.85 ; \mathrm{H}, 5.95 ; \mathrm{N}, 17.98$. Found: $\mathrm{C}, 67.78$; $\mathrm{H}, 6.16 ; \mathrm{N}, 17.91$.

$\mathrm{N}$-(3-Cyano-1-(pyridin-2-yl)-4,5,6,7-tetrahydro- $1 \mathrm{H}$-indol$2-y l)$ acetamide $(3 g)$

It was obtained as a brown solid $\left[\mathrm{EtOH} / \mathrm{H}_{2} \mathrm{O}\right]$. Yield $62 \%$, m.p. $168-170{ }^{\circ} \mathrm{C}$. FT-IR (KBr) $v_{\text {max }} 3300,2300,1730$ $\mathrm{cm}^{-1} .{ }^{1} \mathrm{H}-\mathrm{NMR}$ (DMSO- $\left.d, 300 \mathrm{MHz}\right) \delta=1.7-1.75(\mathrm{~m}, 4 \mathrm{H}, 2$ $\left.\times \mathrm{CH}_{2}\right), 2.4-2.6\left(\mathrm{~m}, 7 \mathrm{H},(4 \mathrm{H}) 2 \times \mathrm{CH}_{2}, 3 \mathrm{H}, \mathrm{CH}_{3}\right), 7.6-8.4(\mathrm{~m}$, $4 \mathrm{H}, \mathrm{Ar}-\mathrm{H}), 9.9$ (s, $1 \mathrm{H}, \mathrm{NH}, \mathrm{D}_{2} \mathrm{O}$ exchangeable) ppm. EIMS $m / z(\%) 280\left[\mathrm{M}^{+}\right](100)$. Anal. Calcd for $\mathrm{C}_{16} \mathrm{H}_{16} \mathrm{~N}_{4} \mathrm{O} ; \mathrm{C}, 68.55$; H, 5.75; N, 19.99. Found: C, 68.54; H, 5.42; N, 19.88 .
N-(3-Cyano-1-phenyl-1,4-dihydroindeno[1,2-b]pyrrol2-yl)acetamide (4a)

It was obtained as a brown solid $\left[\mathrm{MeOH} / \mathrm{H}_{2} \mathrm{O}\right]$. Yield 64\%, m.p. 215-219 ${ }^{\circ} \mathrm{C}$. FT-IR (KBr) $v_{\max } 3450(\mathrm{NH}), 2365$ $(\mathrm{CN}), 1710(\mathrm{C}=\mathrm{O}) \mathrm{cm}^{-1} .{ }^{1} \mathrm{H}-\mathrm{NMR}$ (DMSO- $\left.d, 300 \mathrm{MHz}\right) \delta$ $=2.3\left(\mathrm{~s}, 3 \mathrm{H}, \mathrm{CH}_{3}\right), 3.58\left(\mathrm{~s}, 2 \mathrm{H}, \mathrm{CH}_{2}\right), 7.1-7.67(\mathrm{~m}, 9 \mathrm{H}, \mathrm{Ar}-\mathrm{H})$, 10.47 (br.s, $1 \mathrm{H}, \mathrm{NH}, \mathrm{D}_{2} \mathrm{O}$ exchangeable) ppm. EIMS $\mathrm{m} / \mathrm{z}$ (\%) $313\left[\mathrm{M}^{+}\right]$(8), $146(100)$. Anal. Calcd for $\mathrm{C}_{20} \mathrm{H}_{15} \mathrm{~N}_{3} \mathrm{O}$ : C, 76.66; H, 4.82; N, 13.41. Found: C, 76.69; H, 4.98; N, 13.57.

\section{General methods for the preparation of aryl-pyrrolo [2,3- d]pyrimidin-4-ones 5 and 6}

A suspension of the appropriate compound $\mathbf{1}$ or $\mathbf{2}$ $(0.01 \mathrm{~mol})$ in formic acid $(20 \mathrm{~mL}, 85 \%)$ was refluxed for 3 $\mathrm{h}$, cooled, poured onto ice-water to give a precipitate which was filtered off, dried, and recrystallized from appropriate solvent to afford 5 and 6 .

9-(4-Methoxyphenyl)-5,6,7,8-tetrahydro-3H-pyrimido [4,5-b]indol-4(9H)-one (5b)

It was obtained as a brown solid [EtOH]. Yield 65\%, m.p. $272-276{ }^{\circ} \mathrm{C}$. FT-IR (KBr) $v_{\max } 3230,1690,1560 \mathrm{~cm}^{-1}$. ${ }^{1} \mathrm{H}-\mathrm{NMR}\left(\mathrm{DMSO}-d_{6}, 300 \mathrm{MHz}\right) \delta=1.33-1.58(\mathrm{~m}, 4 \mathrm{H}, 2 \times$ $\left.\mathrm{CH}_{2}\right), 2.2-2.48\left(\mathrm{~m}, 4 \mathrm{H}, 2 \times \mathrm{CH}_{2}\right), 3.52\left(\mathrm{~s}, 3 \mathrm{H}, \mathrm{O}-\mathrm{CH}_{3}\right)$, 6.9-7.5 (m, 4H, Ar-H), 9.3 (s, 1H, $\left.\mathrm{C}_{2}-\mathrm{H}\right), 12.40(\mathrm{~s}, 1 \mathrm{H}, \mathrm{NH}$, $\mathrm{D}_{2} \mathrm{O}$ exchangeable) ppm. EIMS $\mathrm{m} / z(\%) 295\left[\mathrm{M}^{+}\right]$(18.7), 279 (100). Anal. Calcd for $\mathrm{C}_{17} \mathrm{H}_{17} \mathrm{~N}_{3} \mathrm{O}_{2}: \mathrm{C}, 69.14 ; \mathrm{H}, 5.80 ; \mathrm{N}$, 14.23. Found: C, 69.19; H, 5.98; N, 14.53 .

9-o-Tolyl-5,6,7,8-tetrahydro-3H-pyrimido[4,5-b]indol-4 (9H)-one (5d)

It was obtained as a yellowish brown solid $[\mathrm{MeOH}]$. Yield 79\%, m.p. $228-231^{\circ} \mathrm{C}$. FT-IR (KBr) $v_{\max } 3230,1690$, $1560 \mathrm{~cm}^{-1} .{ }^{1} \mathrm{H}-\mathrm{NMR}$ (DMSO- $\left.d, 300 \mathrm{MHz}\right) \delta=1.53-1.78$ $\left(\mathrm{m}, 4 \mathrm{H}, 2 \times \mathrm{CH}_{2}\right), 2.12\left(\mathrm{~s}, 3 \mathrm{H}, \mathrm{CH}_{3}\right), 2.2-2.58(\mathrm{~m}, 4 \mathrm{H}, 2 \times$ $\mathrm{CH}_{2}$ ), 7.2-7.6 (m, 4H, Ar-H), 9.3 (s, $\left.1 \mathrm{H}, \mathrm{C}_{2}-\mathrm{H}\right), 12.18(\mathrm{~s}, 1 \mathrm{H}$, $\mathrm{NH}, \mathrm{D}_{2} \mathrm{O}$ exchangeable) ppm. EIMS $m / z(\%) 279\left[\mathrm{M}^{+}\right](17)$, 118 (100). Anal. Calcd for $\mathrm{C}_{17} \mathrm{H}_{17} \mathrm{~N}_{3} \mathrm{O}: \mathrm{C}, 73.10 ; \mathrm{H}, 6.13 ; \mathrm{N}$, 15.04; O, 5.73. Found: C, 73.39; H, 6.23; N, 15.34; O, 5.94.

9-p-Tolyl-5,6,7,8-tetrahydro-3H-pyrimido[4,5-b]indol-4 (9H)-one (5e)

It was obtained as a brown solid [EtOH]. Yield $71 \%$, m.p. $206-210^{\circ} \mathrm{C}$. FT-IR (KBr) $v_{\max } 3430,3330,1720,1690$, $1560 \mathrm{~cm}^{-1}$. ${ }^{1} \mathrm{H}-\mathrm{NMR}$ (DMSO- $\left.d_{6}, 300 \mathrm{MHz}\right) \delta=1.53-1.78$ $\left(\mathrm{m}, 4 \mathrm{H}, 2 \times \mathrm{CH}_{2}\right), 1.98\left(\mathrm{~s}, 3 \mathrm{H}, \mathrm{CH}_{3}\right), 2.2-2.68(\mathrm{~m}, 4 \mathrm{H}, 2 \times$ $\left.\mathrm{CH}_{2}\right), 7.2-7.8(\mathrm{~m}, 4 \mathrm{H}, \mathrm{Ar}-\mathrm{H}), 9.4\left(\mathrm{~s}, 1 \mathrm{H}, \mathrm{C}_{2}-\mathrm{H}\right), 12.15(\mathrm{~s}$, $1 \mathrm{H}, \mathrm{NH}, \mathrm{D}_{2} \mathrm{O}$ exchangeable) ppm. EIMS $m / z(\%) 279\left[\mathrm{M}^{+}\right]$ (19.3), 188 (100). Anal. Calcd for $\mathrm{C}_{17} \mathrm{H}_{17} \mathrm{~N}_{3} \mathrm{O}$ : C,73.69; $\mathrm{H}$, 6.53; N, 14.32. Found: C, 73.79; H, 6.61; N, 14.19.

9-(1,5-Dimethyl-3-oxo-2-phenyl-2,3-dihydro-1H-pyrazol4-yl)-5,6,7,8-tetrahydro-3H-pyrimido[4,5-b]indol-4(9H)one (5f)

It was obtained as a yellowish brown solid [EtOH]. Yield $83 \%$, m.p. $260-265^{\circ} \mathrm{C}$. FT-IR (KBr) $v_{\max } 3430,3330$, 
$1720,1690,1560 \mathrm{~cm}^{-1} .{ }^{1} \mathrm{H}-\mathrm{NMR}$ (DMSO- $\left.d_{6}, 300 \mathrm{MHz}\right) \delta=$ 1.43-1.68 (m, $\left.4 \mathrm{H}, 2 \times \mathrm{CH}_{2}\right), 2.2-2.45\left(\mathrm{~m}, 4 \mathrm{H}, 2 \times \mathrm{CH}_{2}\right)$, $2.33\left(\mathrm{~s}, 3 \mathrm{H}, \mathrm{CH}_{3}\right), 3.12\left(\mathrm{~s}, 3 \mathrm{H}, \mathrm{N}-\mathrm{CH}_{3}\right), 7.2-7.6(\mathrm{~m}, 5 \mathrm{H}, \mathrm{Ar}-$ $\mathrm{H}), 9.1\left(\mathrm{~s}, 1 \mathrm{H}, \mathrm{C}_{2}-\mathrm{H}\right), 12.3\left(\mathrm{~s}, 1 \mathrm{H}, \mathrm{NH}, \mathrm{D}_{2} \mathrm{O}\right.$ exchangeable) ppm. EIMS $m / z$ (\%) $375\left[\mathrm{M}^{+}\right]$(24.6), 187 (100). Anal. Calcd for $\mathrm{C}_{21} \mathrm{H}_{21} \mathrm{~N}_{5} \mathrm{O}_{2}: \mathrm{C}, 67.18 ; \mathrm{H}, 5.64 ; \mathrm{N}, 18.65$. Found: C, 67.28; H, 5.36; N, 18.36 .

\section{9-(Pyridin-2-yl)-5,6,7,8-tetrahydro-3H-pyrimido[4,5-b]} indol-4(9H)-one $(\mathbf{5 g})$

It was obtained as a brownish red solid [EtOH]. Yield $68 \%$, m.p. $168-170{ }^{\circ} \mathrm{C}$. FT-IR $(\mathrm{KBr}) v_{\max } 3310,1682,1587$ $\mathrm{cm}^{-1} .{ }^{1} \mathrm{H}-\mathrm{NMR}$ (DMSO- $\left.d_{6}, 300 \mathrm{MHz}\right) \delta=1.7-1.75(\mathrm{~m}, 4 \mathrm{H}$, $\left.2 \times \mathrm{CH}_{2}\right), 2.4-2.6\left(\mathrm{~m}, 4 \mathrm{H}, 2 \times \mathrm{CH}_{2}\right), 7.6-8.4(\mathrm{~m}, 4 \mathrm{H}, \mathrm{Ar}-$ $\mathrm{H}), 9.3\left(\mathrm{~s}, 1 \mathrm{H}, \mathrm{C}_{2}-\mathrm{H}\right), 12.21$ (s, $1 \mathrm{H}, \mathrm{NH}, \mathrm{D}_{2} \mathrm{O}$ exchangeable) ppm. EIMS $m / z$ (\%) $266\left[\mathrm{M}^{+}\right]$(16.4), 132 (100). Anal. Calcd for $\mathrm{C}_{15} \mathrm{H}_{14} \mathrm{~N}_{4}$ : C, 67.65; H, 5.30; N, 21.04. Found: C, $67.54 ; \mathrm{H}, 5.62 ; \mathrm{N}, 21.88$.

\section{0-Phenyl-5,10-dihydro-3H-indeno[2', 1':4,5]pyrrolo[2,3-d] pyrimidin-4-one (6a)}

It was obtained as an orange solid $[\mathrm{MeOH}]$. Yield $58 \%$, m.p. $215-218^{\circ} \mathrm{C}$. FT-IR (KBr) $v_{\max } 3330,1705,1590$ $\mathrm{cm}^{-1} \cdot{ }^{1} \mathrm{H}-\mathrm{NMR}$ (DMSO- $\left.d_{6}, 300 \mathrm{MHz}\right) \delta=3.51(\mathrm{~s}, 2 \mathrm{H}$, $\left.\mathrm{CH}_{2}\right), 7.3-7.8(\mathrm{~m}, 10 \mathrm{H}, \mathrm{Ar}-\mathrm{H}+$ pym $\mathrm{H}), 12.21(\mathrm{~s}, 1 \mathrm{H}, \mathrm{NH}$, $\mathrm{D}_{2} \mathrm{O}$ exchangeable) ppm. EIMS $\mathrm{m} / z$ (\%) $299\left[\mathrm{M}^{+}\right]$(18), 221(100). $\mathrm{C}_{19} \mathrm{H}_{13} \mathrm{~N}_{3} \mathrm{O}$ (299.33). Anal. Calcd for $\mathrm{C}_{19} \mathrm{H}_{13} \mathrm{~N}_{3} \mathrm{O}$ : C, 76.24; H, 4.38; N, 14.04. Found: C, 76.57; H, 4.61; N, 14.53 .

\section{Biological Assay}

\section{1. Anti-inflammatory Activity}

\section{Animals}

Ninety adult male Sprague-Dawley rats (5 rats per group for 14 tested compounds, control (injected with 1 $\mathrm{mL}$ DMSO only, 2 standard drugs), weighing 120-150 g, were housed in cages in a temperature-controlled (25 \pm 1 ${ }^{\circ} \mathrm{C}$ ) environment and provided free access to pelleted food and purified drinking water ad libitum. The protocol of the study was approved by the animal ethics committee of the Faculty of Pharmacy, Helwan University on 10-01-2012. The study was conducted in accordance with the EC. DFT -IRective 86/609/EEC for animal experiments.

\section{Assessment of Anti-inflammatory Activity}

Rat paw oedema assay was carried out according to Winter et al. ${ }^{45}$ Prepared compounds (equimolar to active dose of the reference drug), control and 2 standard drugs were dissolved in $1 \mathrm{~mL}$ DMSO and administrated subcutaneously. One hour later, paw oedema was induced by sub -plantar injection of $0.1 \mathrm{~mL}$ of $1 \%$ carrageenan (Sigma-Aldrich, St. Louis, USA) into the right paw. Paw volume was measured using a water plethysmometer (Basile, Comerio, Italy). The difference between the right and left paw volu- me was measured at 1,2, 3 and $4 \mathrm{~h}$ after induction of inflammation. Control group received $1 \mathrm{~mL}$ DMSO (as to evaluate the interference of DMSO itself in biological test) subcutaneously and carrageenan in sub-plantar region. Results were expressed as percentage inhibition of inflammation. Ibuprofen $(70 \mathrm{mg} / \mathrm{kg})$ and indomethacin $(20$ $\mathrm{mg} / \mathrm{kg}$ ) were used as the reference drugs.

\section{Statistical Analysis}

Results were expressed as the mean \pm SEM, and different groups were compared using one way analysis of variance (ANOVA) followed by Tukey-Kramer test for multiple comparisons, using Graph Pad Instant (version 3.05) as the statistical software.

Calculation: equimolar doses of tested compounds were calculated in relation to these of reference drug: swel $=$ mean difference in rat paw volume between right and left paw \pm SE. \% inhibition $=(1-\mathrm{rt} / \mathrm{rc}) \times 100$ [rt $=$ swel of tested group; $\mathrm{rc}=$ swel of control group].

\section{2. Molecular Docking Study}

\section{MOE 2013.08 Docking}

The molecular docking studies were done using MOE 2013.08 and Leadit 2.1.2. All compounds were built and saved as MOE. Rigid receptor was used as a docking protocol. Both receptor-solvent were kept as a »receptor «. Triangle matcher was used as a placement method. Two rescoring were computed, rescoring 1 was selected as London dG. Rescoring 2 was selected as affinity. The force field was used as a refinement.

\section{Leadit 2.1.2 Docking}

All compounds were built and saved as Mol2. The crystal structure of COX-2 enzyme complexes with indomethacin was downloaded from protein data bank (PDB: 4COX). The protein was loaded into Leadit 2.1.2 and the receptor components were chosen by selection of chain $\mathrm{A}$ as the main chain when complexes with indomethacin. Binding site was defined by choosing indomethacin as the reference ligand to which all coordinates were computed. Amino acids within radius $6.5 \AA$ were selected in the binding site. All chemical ambiguities of residues were left as default. Ligand binding was driven by enthalpy (classic Triangle matching). For scoring, all default settings were restored. Intra-ligand clashes were computed by using clash factor $=0.6$. Maximum number of solutions per iteration $=$ 200. Maximum of solution per fragmentation $=200$. The base placement method was used as the docking strategy.

\section{Result and Discussion}

\section{1. Chemistry}

The availability of $a$-amino ketones is key to the preparation of $o$-amino- cyanopyrroles. ${ }^{46-48}$ Research in- 
dicated that $\alpha$-amino ketones used for the preparation of $o$-amino-cyanopyrroles were usually obtained in situ ${ }^{49-51}$ via the reaction of a-hydroxy ketones with amines in acid medium, ${ }^{52-55}$ or via the reaction of $\alpha$-halo ketones with either amines and/or $\alpha$-amino acids.$^{56}$ As previously mentioned, $a$-hydroxy ketones and $a$-halo ketones, malononitriles or suitable substituted alkylidenemalonitrile and primary amines constituted essential components for the synthesis of $o$-amino-cyanopyrrole derivatives. ${ }^{44,57,58}$

Regarding fused pyrrole and fused pyrrolopyrimidine derivatives there are few studies reporting the synthesis of $o$-aminocyano-tetrahydrobenzo[b]pyrrole and $o$-aminocyano-octahydroindeno[2,1- $b]$ pyrrole derivatives. Literature also revealed that the reaction of $a$-hydroxycyclohexanone (in place of the a-chloro analogue) with certain amines and malononitrile successfully afforded some $o$-aminocyanotetrahydrobenzo[b]pyrroles but in poor yields (20-30\%). ${ }^{44,58,59}$ These results also indicated that some side reactions happened (Fig. 2a).

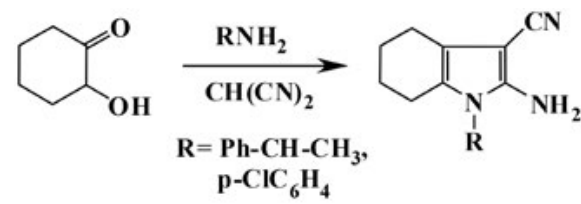

Figure 2a. Typical synthesis of tetrahydrobenzo[ $b]$ pyrroles
Attempts made to develop such compounds using the regular reactants ( $\alpha$-chlorocyclohexanone and/or 2-chloroindenone, with certain aromatic amines and malononitrile) were unfruitful. ${ }^{60}$ We also found that a-chlorocyclohexanone under the reaction conditions $\mathrm{EtOH} / \mathrm{NaOH}$ (polar basic medium) might undergo what is called Favorskii rearrangement, ${ }^{61}$ rearrangement of an a-halo ketone upon treatment with a base; the reaction continues through cyclopropanone intermediate formed by nucleophilic attack [ $\left.\mathrm{EtO}^{-}\right]$. In addition to the possibility of condensation with amine to give the anil (Fig. 2b). ${ }^{62,63}$

Taking the previous results into consideration, we tried to diminish the potential of such side reactions. Our plan was to prepare the novel intermediate 2-(2-oxocyclohexyl) malononitrile (I) from the reaction of 2-chlorocyclohexanone with malononitrile in a nonpolar solvent, followed by condensation with the appropriate aromatic amines.

By applying this method, we successfully set up the required tetrahydrobenzo[b]pyrroles 1 with a fair yield. Using the same conditions with 2-chloroindenone it produced 2 (Scheme 1).

On the other hand, aminocyano pyrrole derivatives 1 and $\mathbf{2}$ were converted to the corresponding acetylated derivatives 3 and 4 via condensation with acetic anhydyride. $\left.{ }^{64-66} 3-\mathrm{d}\right]$ pyrimidines were reported to act as potent anticancer agents, in this work, a series of novel 2-substituted-

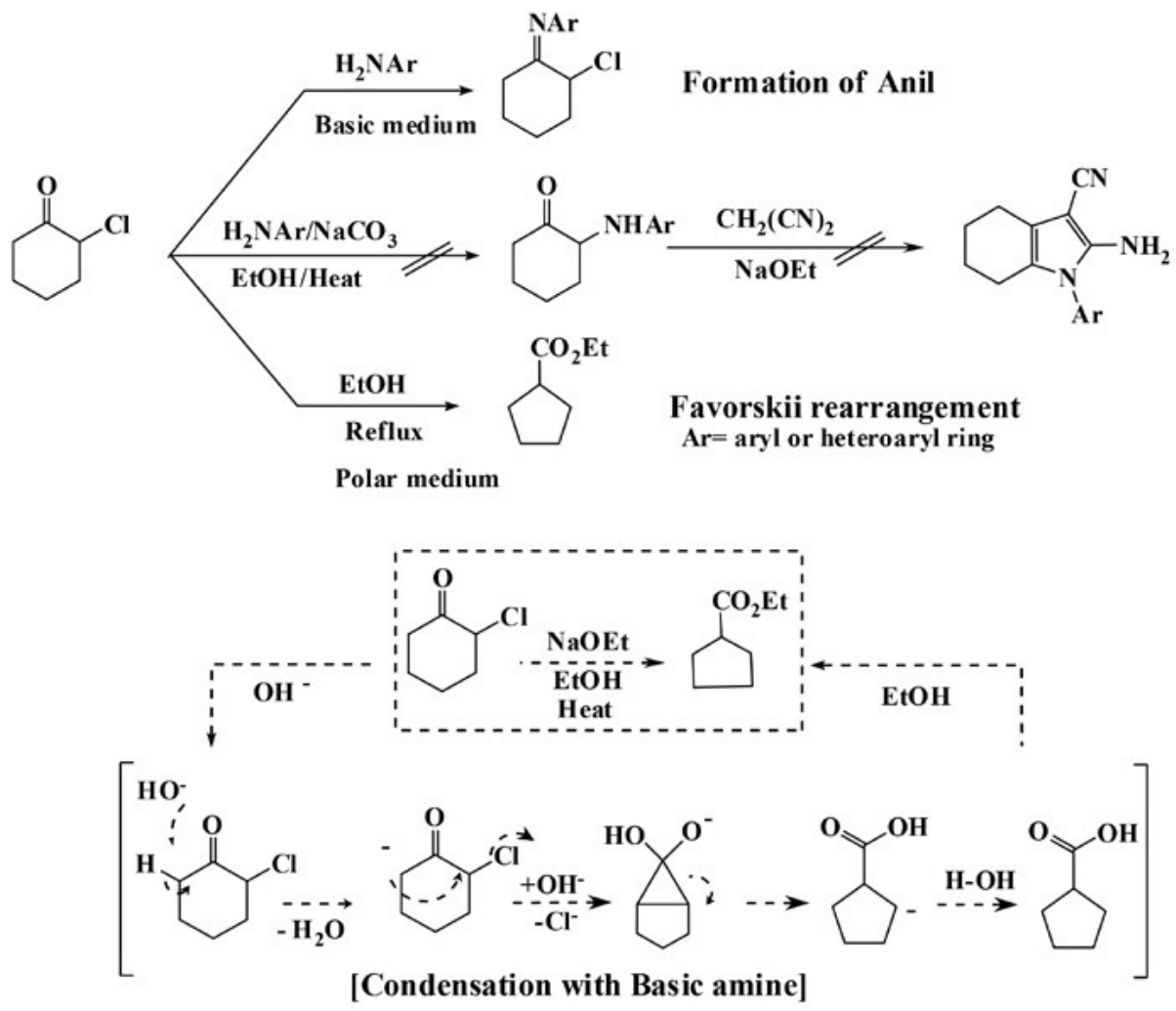

Figure 2b. Favoriskii rearrangement (polar basic medium rearrangement), ${ }^{57}$ and anil formation ${ }^{58,59}$ 

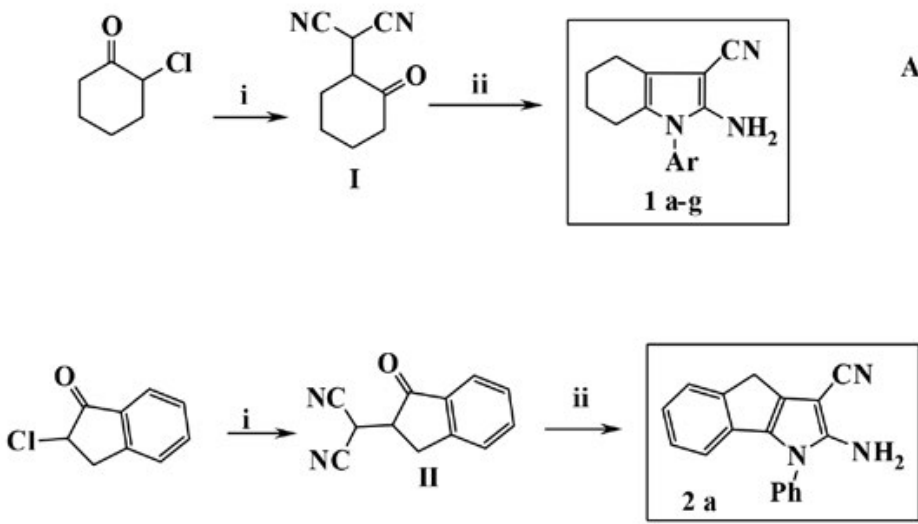<smiles>COc1ccccc1-c1ccccc1</smiles>

$\mathrm{i}=\underset{\mathrm{NaOH} / \mathrm{CHCl}_{3}}{\mathrm{NCCH}_{2} \mathrm{CN}} \quad \mathrm{ii}=\underset{\mathrm{EtOH} / \mathrm{HCl}}{\mathrm{ArNH}_{2}}$

Scheme 1: Synthesis of tetrahydroindoles $\mathbf{1 a}-\mathbf{g}$ and $\mathbf{2 a}$

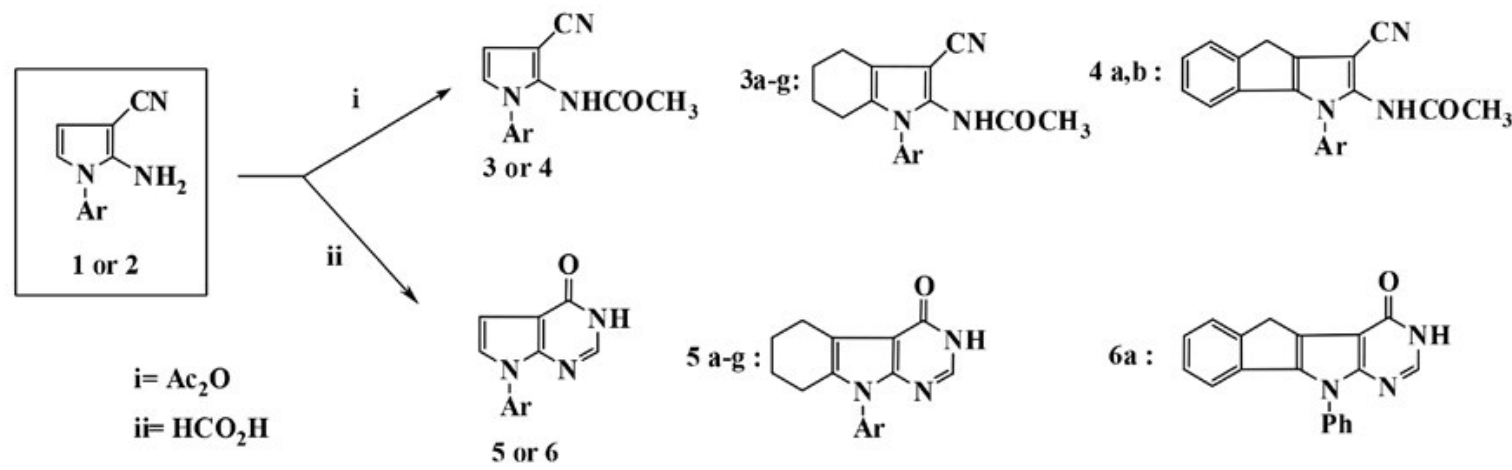

Scheme 2: Synthesis of acetylated pyrroles $3 \mathbf{a}-\mathbf{g}, \mathbf{4 a}, \mathbf{b}$ and pyrrolopyrimidines $5 \mathbf{a}-\mathbf{g}, \mathbf{6 a}$

3-cyano-4-phenyl-pyrrole 5, 6, 11-18, and 5-phenyl-pyrrolo[2,3-d]pyrimidine derivatives 7-10, 19-24 bearing either sulfathiazole or sulfapyridine were synthesized. The structures of these compounds were confirmed by elemental analysis, IR, (1 Compounds $\mathbf{1}$ and $\mathbf{2}$ were converted to pyrrolo[2,3- $d]$ pyrimidine-4-ones 5 and $\mathbf{6}$ via condensation with formic acid (Scheme 2). ${ }^{64}$

\section{2. Biological Results and Discussion}

Fourteen of the synthesized compounds were evaluated for their anti-inflammatory activity, using a method established by Harrk et al. ${ }^{31} 4$-benzodioxine or pyrrole nucleus are described. All the newly synthesized compounds were examined for their in vitro and in vivo anti-inflammatory activity. Several derivatives, including (S Five of the tested compounds induced significant anti-inflammatory activity, compared with that of ibuprofen and indomethacin. Compound $\mathbf{1 b}$ exerted significant activities compared to standard drugs at all time intervals post-carrageenan $\left(\cong 78 \%, \cong 80 \%, \cong 84 \%\right.$ and $\cong 85 \%$ inhibition at $1^{\text {st }}$, $2^{\text {nd }}, 3^{\text {rd }}$ and $4^{\text {th }}$ hour interval post-carrageenan). The activity profile was the same as for standard drugs (response increasing with time). Compound $1 \mathrm{c}$ exerted noticeable acti- vities compared to standard drugs at the $1^{\text {st }}$ and $2^{\text {nd }}$ hour post-carrageenan $\left(\cong 81 \%\right.$ and $\cong 82 \%$ inhibition at $1^{\text {st }}$ and $2^{\text {nd }}$ hour interval post-carrageenan). The activity profile was the same as standard drugs (response increasing with time), yet the activity showed weak, yet significant activities, decreasing at $3^{\text {rd }}$ and $4^{\text {th }}$ hour post-carrageenan $\cong 79 \%$ and $\cong 75 \%$ inhibition at $3^{\text {rd }}$ and $4^{\text {th }}$ hour interval post-carrageenan). Compounds $\mathbf{1 d}$ and $\mathbf{5 b}$ showed a marked anti -inflammatory effect than standard drugs, from the $1^{\text {st }}$ hour to $4^{\text {th }}$ hour post-carrageenan. Compound $\mathbf{5 b}$ showed a moderate inhibitory action at the $4^{\text {th }}$ hour interval: $76 \%$ inhibition. Yet, compound 1d showed the unusual profile compared to standard drugs: it showed $\cong 79 \%$ inhibition at $2^{\text {nd }}$ hour post-carrageenan and $\cong 70 \%$ inhibition at $3^{\text {rd }}$ hour post-carrageenan and then decreased to $66 \%$ inhibition at $4^{\text {th }}$ hour post-carrageenan. Compound $1 \mathrm{~g}$ exerted a moderate activity compared to standard drugs at the $3^{\text {rd }}$ and $4^{\text {th }}$ hour post-carrageenan (\% inhibition $\cong 48$ at $3^{\text {rd }}$ hour and $61 \%$ at $4^{\text {th }}$ hour post-carrageenan), it showed no activity at $1^{\text {st }}$ and $2^{\text {nd }}$ hour intervals post-carrageenan injection. Compounds 1f, 3c, 3d, 3e, $\mathbf{3 f}$ and $\mathbf{5 d}, \mathbf{e}$ were all inactive over all tested periods, showing \% inhibition $<12,21,25$ and 38 at $1^{\text {st }}$ to $4^{\text {th }}$ hours, respectively, and were indicated as inactive in Table 1. 
Table 1. In vivo anti-inflammatory activity results for active compounds.

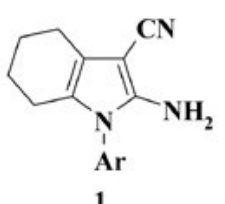<smiles>[14CH2]n1c2c(c3c(=O)[nH]cnc31)CCCC2</smiles>

\begin{tabular}{|c|c|c|c|c|c|c|c|c|}
\hline \multirow{3}{*}{$\begin{array}{l}\text { Compounds } \\
/ /(\mathbf{A r}=)\end{array}$} & \multicolumn{8}{|c|}{ Oedema induced by carrageenan (\% Oedema inhibition relative to control) } \\
\hline & \multicolumn{2}{|c|}{$1 \mathrm{Hr}$} & \multicolumn{2}{|c|}{$2 \mathrm{Hr}$} & \multicolumn{2}{|c|}{$3 \mathrm{Hr}$} & \multicolumn{2}{|c|}{$4 \mathrm{Hr}$} \\
\hline & Swel \pm SE & Swel \pm SE & $\%$ inh & Swel \pm SE & $\%$ inh & Swel \pm SE & $\%$ inh & $\%$ inh \\
\hline & $0.048 \pm 0.024^{\mathrm{a}}$ & 78.7 & $0.052 \pm 0.02^{\mathrm{a}}$ & 80 & $0.086 \pm 0.048^{\mathrm{a}}$ & 84.3 & $0.098 \pm 0.04^{\mathrm{a}}$ & 84.69 \\
\hline c & $0.042 \pm 0.008^{\mathrm{a}}$ & 81.37 & $0.046 \pm 0.009^{\mathrm{a}}$ & 82.3 & $0.116 \pm 0.048^{\mathrm{a}}$ & 79.6 & $0.159 \pm 0.029^{\mathrm{a}}$ & 75.06 \\
\hline 1 & $0.19 \pm 0.036$ & 15.9 & $0.076 \pm 0.038^{\mathrm{a}}$ & 70.76 & $0.022 \pm 0.005^{\mathrm{a}}$ & 95.98 & $0.048 \pm 0.007^{\mathrm{a}}$ & 92 \\
\hline & $0.2137 \pm 0.03$ & 6.35 & $0.054 \pm 0.01^{\mathrm{a}}$ & 78.16 & $0.086 \pm 0.018^{a}$ & 69.43 & $0.083 \pm 0.068^{\mathrm{a}}$ & 66.73 \\
\hline & $0.2242 \pm 0.031$ & 0.79 & $0.25 \pm 0.007$ & 3.65 & $0.284 \pm 0.04$ & 48.17 & $0.251 \pm 0.031^{\mathrm{a}}$ & 60.74 \\
\hline $5 b$ & $0.152 \pm 0.016$ & 32.74 & $0.15 \pm 0.024$ & 42.3 & $0.234 \pm 0.04^{\mathrm{a}}$ & 57.29 & $0.152 \pm 0.057^{\mathrm{a}}$ & 76.25 \\
\hline Indomethacin & $0.224 \pm 0.004$ & 0.88 & $0.764 \pm 0.009$ & 20.7 & $0.286 \pm 0.004$ & 44.65 & $0.084 \pm 0.01$ & a 78.58 \\
\hline Ibuprofen & $0.216 \pm 0.033$ & 4.42 & $0.158 \pm 0.04$ & 39.23 & $0.286 \pm 0.008$ & 48.175 & $0.193 \pm 0.007^{\mathrm{a}}$ & 69.84 \\
\hline Control & $0.228 \pm 0.027$ & & $0.26 \pm 0.037$ & & $0.548 \pm 0.08$ & & $0.64 \pm 0.038$ & \\
\hline
\end{tabular}

swel $=$ mean difference in rat paw volume between right and left paw. $\pm \mathrm{SE}$ a: significantly different from control at the same time interval at $\mathrm{p}<0.05 \%$ inhibition $=(1-\mathrm{rt} / \mathrm{rc}) \times 100 \quad[\mathrm{rt}=$ swel of tested group; $\mathrm{rc}=$ swel of control group $]$ swel $=$ swelling $\mathrm{SE}=$ standard error $\%$ inh $=\%$ inhibition

\section{3. Molecular Docking Results}

Molecular modeling has become very important in the discovery and design of new agents. ${ }^{67-70} 3 \mathrm{D}-\mathrm{QSAR}$ and

a)

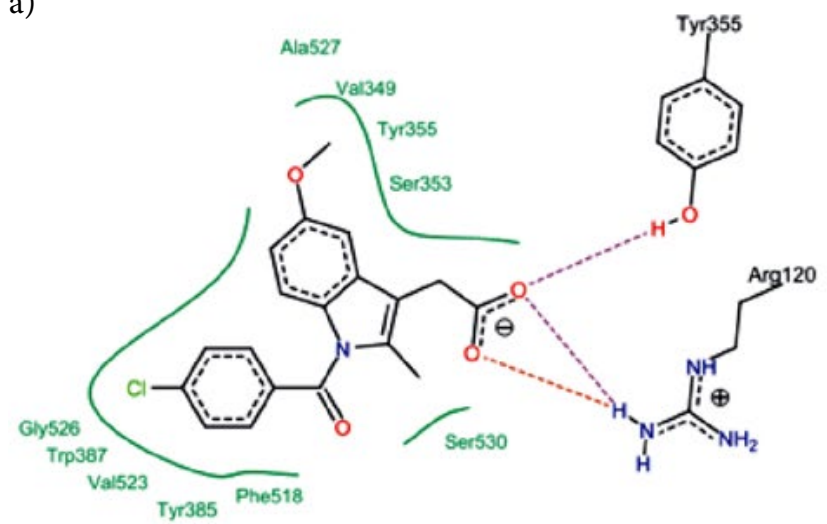

docking studies were carried out on 23 pyrrole derivatives, to model their HIV-1 gp41 inhibitory activities. The 2D, 3D-QSAR studies were performed using CODESSA software package and comparative molecular field analysis

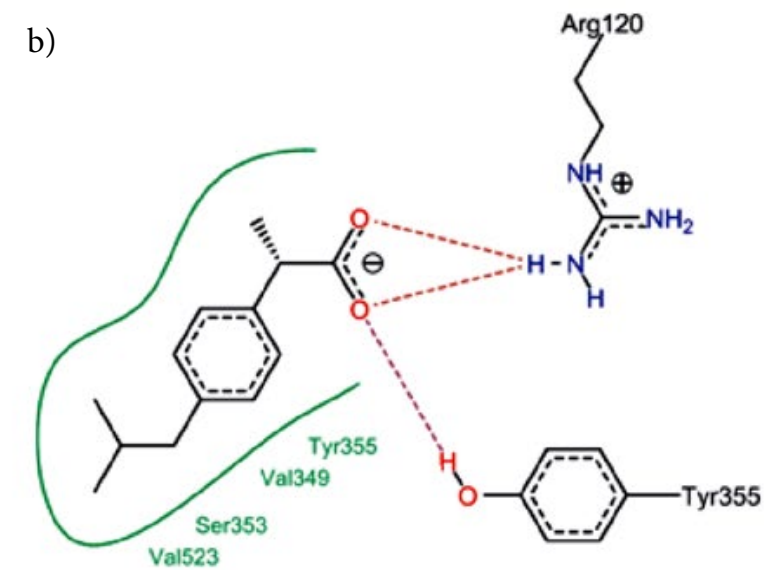

Figure 3a. Binding modes of both A) indomethacin and B) ibuprofen. This was computed with Leadit 2.1.2 
(CoMFA It also helps in the interpretation and explanation of the biological results. Molecular docking is one of these approaches and is used to predict the binding mode of organic compounds. ${ }^{68} \mathrm{~A}$ molecular docking study had been done using both MOE 2013.08 ${ }^{71}$ and Leadit 2.1.2 software. ${ }^{72,73}$ Possible binding modes of the active compounds inside the active site of COX-2 were estimated. Indomethacin and ibuprofen were also docked. The presence of a carboxylic group in both indomethacin and ibuprofen was important for the carboxylate anion to form an electrostatic interaction with the cationic guanidine moiety of Arg 120 residue found in the active site of the COX-2 enzyme. The oxygen atom of carbonyl group found in this carboxylic moiety participated with the formation of a hydrogen bond with the hydrogen atom of -OH group of Tyr 355 residue. The binding affinity of indomethacin and ibuprofen was found to be $-30.24 \mathrm{kcal} / \mathrm{mol}$ and $-19.09 \mathrm{kcal} / \mathrm{mol}$, respectively (Fig. 3a).
The clash score was computed with Leadit 2.1.2 software indicating that both drugs have a low clash score (Table 2).

The presence of the $p$-chloro group in the derivative 1c with higher lipophilic contribution value $(-10.90)$ improving the binding modes and interactions, compared with other tested compounds. All compounds with 2 -amino-(substituted)- $1 \mathrm{H}$-indole-3-carbonitrile shared a hydrogen bond formed between their nitrile groups and the $\mathrm{OH}$ group of Tyr 355. Compound $\mathbf{1 g}$ showed a mode of binding in which three hydrogen bonds with Tyr 355, Arg 120 and Ser 530 were formed. Finally, compound $\mathbf{5 b}$ showed three hydrogen bonds as well with His 90, Tyr 355 and Ser 530 (Fig. 3b).

Compounds $1 \mathbf{d}$ and $\mathbf{1 g}$ had the highest clash penalty score (11.54) which affected their fitting in the binding site and resulted in the lowest affinity values in both MOE 2013.08 and Leadit 2.1.2 docking results. That could expla-

Table 2. The clash score for active compounds and standard drugs using Leadit 2.1.2 software.

\begin{tabular}{lccrr}
\hline $\begin{array}{l}\text { Compound/ } \\
\text { Standard drugs }\end{array}$ & $\begin{array}{c}\text { \% of } \\
\text { inhibition }\end{array}$ & $\begin{array}{c}\text { MOE } \\
\text { docking score }\end{array}$ & $\begin{array}{c}\text { Leadit docking } \\
\text { Docking score } \\
\text { (kcal/mol) }\end{array}$ & $\begin{array}{c}\text { Lipo score } \\
\text { Clash }\end{array}$ \\
\hline 1b & 84.69 & -11.68 & -17.03 & -13.84 \\
Indomethacin & 78.58 & -15.25 & -21.24 & -12.93 \\
5b & 76.25 & -10.38 & -15.47 & -12.97 \\
1c & 75.06 & -10.52 & -17.23 & -10.90 \\
Ibuprofen & 69.84 & -13.35 & -19.09 & -10.41 \\
1d & 66.73 & -10.24 & -16.02 & -11.42 \\
1g & 60.73 & -10.04 & -12.54 & -14.06 \\
\hline
\end{tabular}

a)

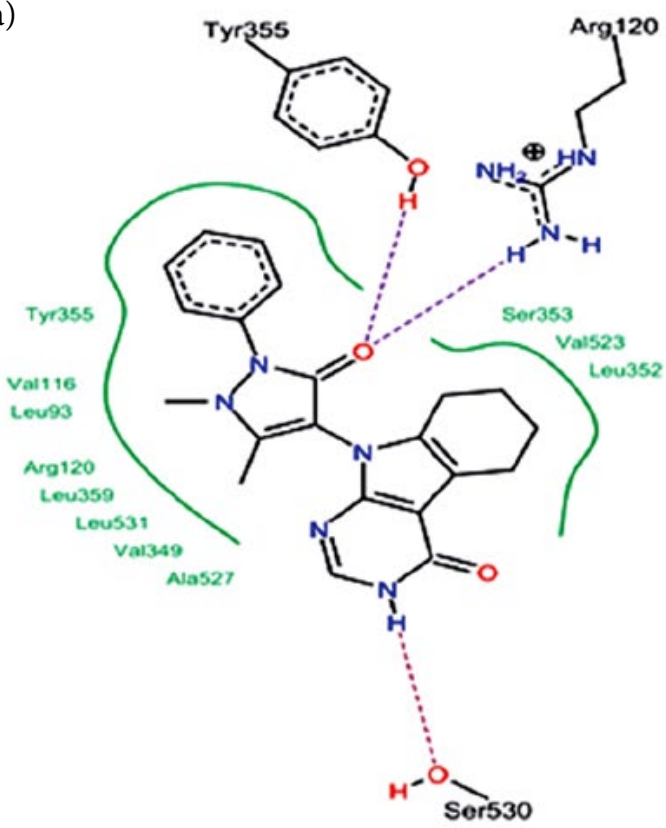

b)

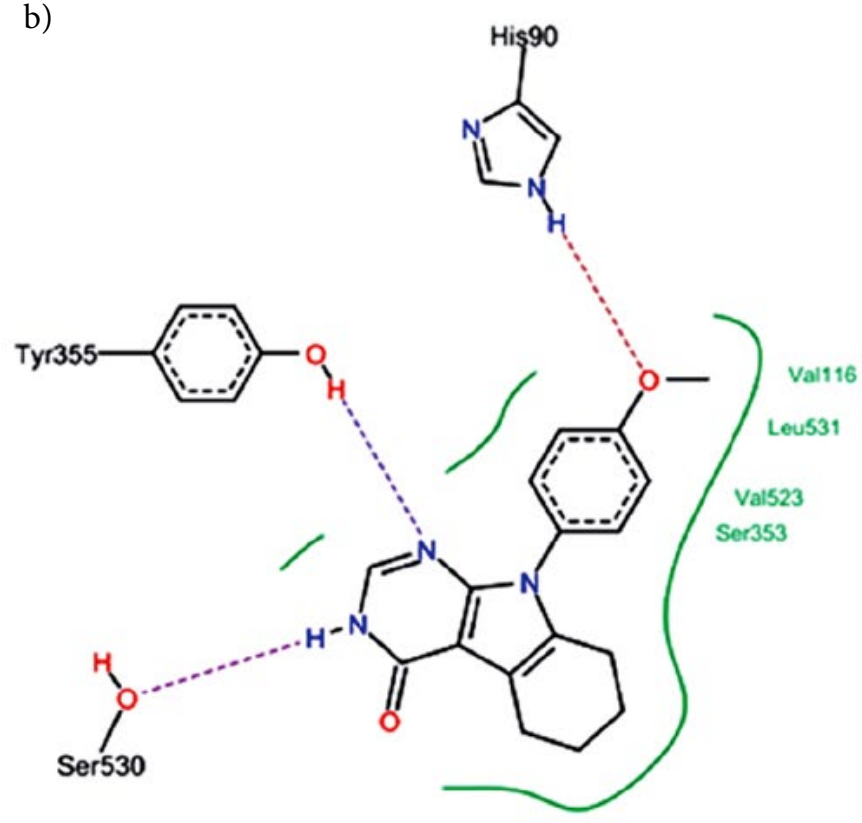

Figure 3b. A) Compound $\mathbf{1 g}$ possible binding mode inside COX-2. B) Compound $\mathbf{5 b}$ predicted interactions. 
in their lower inhibition activity compared to the other compounds.

\section{4. Structure-activity Relationships (SAR)}

To investigate SAR of any NSAIDs, pathophysiology of inflammation and its treatment have to be well understood. Inflammation represents the response to injury. ${ }^{74}$ Many processes are involved in the promotion of the inflammatory process, one of them is the secretion of arachidonic acid (AA) from damaged cells membranes. AA is metabolized by enzyme COX into prostanoids (as prostaglandins) and lipoxygenase (leukotrienes). ${ }^{75}$ The major mechanism of action of NSAIDs was found to be the inhibition of PG synthesis, through inhibition of COXs, that is to say preventing the AA from forming $P G{ }^{76-78}$ it has a very short half-live in blood, its oxidization to 15-ketoprostaglandins is catalyzed by 15 -hydroxyprostaglandin dehydrogenase (15-PGDH

In order to design any structure with pyrrole moiety or its fused form indole, vital considerations must be taken to ensure its anti-inflammatory activity. ${ }^{32,74,79,80}$

First, the structure should consist of an acidic moiety (carboxylic acid, enols, ester etc.) attached to a planar, aromatic functional group (appears to correlate with the double bond of AA), ${ }^{31} 4$-benzodioxine or pyrrole nucleus are described. All the newly synthesized compounds were examined for their in vitro and in vivo anti-inflammatory activity. Several derivatives, including (S and a polar linking group (which attaches the aromatic ring to a lipophilic group in AA).$^{30}$ Addition of a second hydrophobic ring, not coplanar with the original aromatic ring, was found to enhance activity, ${ }^{35}$ this second heteroaromatic ring or heterocyclic ring was believed to provide the necessary geometry to attach to AA. ${ }^{81}$ Taking indomethacin (benzo $[b]$ pyrrole) as an example, it was found that $N$-benzoyl moiety seems to play an important role for the COX-1 activity of indomethacin. ${ }^{32}$ If the latter moiety is replaced with any other bulkier $\mathrm{N}$-substituents, efficient binding to COX-1 pocket is prevented. ${ }^{12,82-84}$

Taking all this into consideration, and to analyze our SAR, two structural components were considered: the nature of the heterocycle nucleus and the character of the side chain ( $N$-substitution). First, the influence of the nature of the aromatic heterocyclic system: fused pyrrole 1a, 1c, and 1d showed the highest activity over fused pyrrolopyrimidine $\mathbf{5 b}$. Regarding the side chain type, addition of bulky heterocyclic ring (anti-pyrine) in compound $1 \mathrm{~g}$ causes the activity to decrease over the substituted phenyl in compounds 1a, 1c, and 1d. Also methoxy group at para-position in compound 1a has conferred significantly higher activity during all time intervals than the methyl goup at ortho-position in compound 1d. Replacing the antipyrine moiety in compound $\mathbf{1 g}$ with the halogen group at para-position in compound $1 \mathrm{c}$ has conferred significantly higher activity during $1^{\text {st }}$ and $2^{\text {nd }}$ hour time intervals, with decreased activity in $3^{\text {rd }}$ and $4^{\text {th }}$ hour post-carrageenan.

\section{Conclusion}

A new strategy was developed to prepare a series of 2-amino-1-(aryl)-4,5,6,7-tetrahydro- $1 \mathrm{H}$-indole-3-carbonitriles 1a-g and 2-amino-1-(aryl)-4,5,6,7-tetrahydro- $1 \mathrm{H}$ -indole-3-carbonitriles $\mathbf{2 a} \mathbf{a}, \mathbf{b}$ as potential anti-inflammatory agents. Based on their structure, we can conclude that the best aromatic nucleus was the pyrrole with a para substituted phenyl and cyclization to prepare pyrrolopyrimidine derivatives, added some anti-inflammatory activity in the heterocyclic system. The molecular docking study provided the interpretation of the biological activities of the active compounds compared to the two reference drugs indomethacin and ibuprofen.

\section{Acknowledgements}

On the occasion of this, we would like to specify Dr. Rehab Kamel, Assistance professor of pharmacology, Faculty of Pharmacy, Helwan University; and Professor Dr. Aymen Goudah. Professor of Pharmacology, Faculty of Veterinary Medicine, Cairo University; with our sincerest gratitude in appreciation of their great assistance, sincere help, valuable advice and all facilities offered during this study. Our Profuse Thanks and Respects.

\section{References}

1. D.-L. Chen, G.-X. Ma, M.-J. He, Y.-Y. Liu, X.-B. Wang, X.-Q. Yang, Helv. Chim. Acta 2016, 99, 742-746.

DOI:10.1002/hlca.201600159

2. H. Li, S. Fan, J. Cheng, P. Zhang, B. Zhong, W. Shi, Molecules 2016, 21, 1-8.

3. S. S. Fatahala, S. Hasabelnaby, A. Goudah, G. I. Mahmoud, Molecules 2017, 22, 1-18.

4. M. Shroff, S. J. Daharwal, Br. Biomed. Bull. 2017, 5, art. no. 299, 7 pp.

5. M. A. Kale, A. S. Narute, T. M. Kalyankar, Clin. Anti-inflamm. Anti-Allergy Drugs 2014, No. 1, 39-44.

6. I. Lessigiarska, A. Nankov, A. Bocheva, I. Pajeva, A. Bijev, Farmaco 2005, 60, 209-218.

DOI:10.1016/j.farmac.2004.11.008

7. A. Abbas, M. M. Naseer, Acta Chim. Slov. 2014, 61, 792-802.

8. A. A. Fadda, K. M. Elattar, J. Biosci. Med. 2015, 3, 114-123.

9. G. M. Cole, S. A. Frautschy, CNS Neurol. Disord. Drug Targets 2010, 9, 140-148. DOI:10.2174/187152710791011991

10. B. O. Villoutreix, G. Laconde, D. Lagorce, P. Martineau, M. A. Miteva, P. Dariavach, PLoS One 2011, 6, e21117.

DOI:10.1371/journal.pone.0021117

11. J. S. Bang, D. H. Oh, H. M. Choi, B.-J. Sur, S.-J. Lim, J. Y. Kim, 
H.-I. Yang, M. C. Yoo, D.-H. Hahm, K. S. Kim, Arthritis Res. Ther. 2009, 11, R49. DOI:10.1186/ar2662

12. K. D. Rainsford, Inflamm. Pathog. Chronic Dis. 2007, 42, 3-27. DOI:10.1007/1-4020-5688-5_1

13. A. O. H. El-Nezhawy, S. T. Gaballah, M. A. A. Radwan, A. R. B. Abdel-Salam, Med. Chem. (Los Angeles). 2009, No. 202, 558-569.

14. R. Mohareb, F. Al-Omran, M. Abdelaziz, R. Ibrahim, Acta Chim. Slov. 2017, 64, 349-364. DOI:10.17344/acsi.2017.3200

15. S. S. Fatahala, M. S. Mohamed, M. Youns, R. H. A.-E. Hameed, Anticancer. Agents Med. Chem. 2017, 17, 1-12. DOI:10.2174/1871520617666170102152928

16. S. Fatahala, E. Shalaby, S. Kassab, M. Mohamed, Anticancer. Agents Med. Chem. 2015, 15, 517-526.

DOI:10.2174/1871520615666150105113946

17. S. D. Joshi, U. A. More, S. R. Dixit, H. H. Korat, T. M. Aminabhavi, A. M. Badiger, Med. Chem. Res. 2013, 23, 1123-1147. DOI:10.1007/s00044-013-0709-y

18. J. A. Pfefferkorn, Y. Song, K.-L. Sun, S. R. Miller, B. K. Trivedi, C. Choi, R. J. Sorenson, L. D. Bratton, P. C. Unangst, S. D. Larsen, T.-J. Poel, X.-M. Cheng, C. Lee, N. Erasga, B. Auerbach, V. Askew, L. Dillon, J. C. Hanselman, Z. Lin, G. Lu, A. Robertson, K. Olsen, T. Mertz, C. Sekerke, A. Pavlovsky, M. S. Harris, G. Bainbridge, N. Caspers, H. Chen, M. Eberstadt, Bioorg. Med. Chem. Lett. 2007, 17, 4538-4544.

DOI:10.1016/j.bmcl.2007.05.096

19. S. Y. Kang, E.-J. Park, W.-K. Park, H. J. Kim, D. Jeong, M. E. Jung, K.-S. Song, S. H. Lee, H. J. Seo, M. J. Kim, M. Lee, H.-K. Han, E.-J. Son, A. N. Pae, J. Kim, J. Lee, Bioorg. Med. Chem. Lett. 2010, 20, 1705-1711. DOI:10.1016/j.bmcl.2010.01.093

20. X. Teng, H. Keys, J. Yuan, A. Degterev, G. D. Cuny, Bioorg. Med. Chem. Lett. 2008, 18, 3219-3223.

DOI:10.1016/j.bmcl.2008.04.048

21. N. Danchev, A. Bijev, D. Yaneva, S. Vladimirova, I. Nikolova, Arch. Pharm. (Weinheim) 2006, 339, 670-674.

DOI:10.1002/ardp.200600116

22. N. Amishiro, A. Okamoto, C. Murakata, T. Tamaoki, M. Okabe, H. Saito, J. Med. Chem. 1999, 42, 2946-2960.

DOI:10.1021/jm990094r

23. S. B. Almasaudi, N. A. El-Shitany, A. T. Abbas, U. A. Abdel-Dayem, S. S. Ali, S. K. Al Jaouni, S. Harakeh, Oxid. Med. Cell. Longev. 2016, 2016, 1-10.

24. S. Yang, Y. Kim, D. Jeong, J. H. Kim, S. Kim, Y. Son, B. C. Yoo, E. J. Jeong, T. W. Kim, I. H. Lee, J. Y. Cho, Biomol. Ther. 2016, 24, 595-603. DOI:10.4062/biomolther.2016.027

25. W. B. Han, A. H. Zhang, X. Z. Deng, X. Lei, R. X. Tan, Org. Lett. 2016, 18, 1816-1819. DOI:10.1021/acs.orglett.6b00549

26. X. T. Xu, X.-Q. Mou, Q.-M. Xi, W.-T. Liu, W.-F. Liu, Z.-J. Sheng, X. Zheng, K. Zhang, Z.-Y. Du, S.-Q. Zhao, S.-H. Wang, Bioorg. Med. Chem. Lett. 2016, 26, 5334-5339.

DOI:10.1016/j.bmcl.2016.09.034

27. J. L. Amorim, D. L. R. Simas, M. M. G. Pinheiro, D. S. A. Moreno, C. S. Alviano, A. J. R. Da Silva, P. D. Fernandes, PLoS One 2016, 11, 1-18. DOI:10.1371/journal.pone.0153643
28. M. Malmsten, Curr. Top. Med. Chem. 2016, 16, 16-24. DOI:10.2174/1568026615666150703121518

29. A. Bocheva, A. Bijev, A. Nankov, Arch. Pharm. (Weinheim) 2006, 339, 141-144. DOI:10.1002/ardp.200500191

30. M. T. Sarg, M. M. Koraa, A. H. Bayoumi, S. M. Abd El Gilil, Open J. Med. Chem. 2015, 5, 49-96.

DOI:10.4236/ojmc.2015.54005

31. Y. Harrak, G. Rosell, G. Daidone, S. Plescia, D. Schillaci, M. D. Pujol, Bioorg. Med. Chem. 2007, 15, 4876-4890.

DOI:10.1016/j.bmc.2007.04.050

32. G. Dannhardt, W. Kiefer, G. Krämer, S. Maehrlein, U. Nowe, B. Fiebich, Eur. J. Med. Chem. 2000, 35, 499-510. DOI:10.1016/S0223-5234(00)00150-1

33. S. Ushiyama, T. Yamada, Y. Murakami, S. Kumakura, S. Inoue, K. Suzuki, A. Nakao, A. Kawara, T. Kimura, Eur. J. Pharmacol. 2008, 578, 76-86. DOI:10.1016/j.ejphar.2007.08.034

34. G. A. Elmegeed, A. R. Baiuomy, O. M. E. Abdel-Salam, Eur. J. Med. Chem. 2007, 42, 1285-1292.

DOI:10.1016/j.ejmech.2007.01.027

35. J. T. Moon, J. Y. Jeon, H. A. Park, Y.-S. Noh, K.-T. Lee, J. Kim, D. J. Choo, J. Y. Lee, Bioorg. Med. Chem. Lett. 2010, 20, 734737. DOI:10.1016/j.bmcl.2009.11.067

36. M. S. Mohamed, R. Kamel, R. H. Abd El-hameed, Med. Chem. Res. 2012, 22, 2244-2252. DOI:10.1007/s00044-012-0217-5

37. P. N. Praveen Rao, E. E. Knaus, J. Pharm. Pharm. Sci. 2008, 11, 81-110. DOI:10.18433/J3T886

38. L. Gasparini, E. Ongini, G. Wenk, J. Neurochem. 2004, 91, 521-536. DOI:10.1111/j.1471-4159.2004.02743.x

39. A. Carbone, B. Parrino, P. Barraja, V. Spanò, G. Cirrincione, P. Diana, A. Maier, G. Kelter, H.-H. Fiebig, Mar. Drugs 2013, 11, 643-654. DOI:10.3390/md11030643

40. M. S. Mohamed, A. E. Rashad, M. E. A. Zaki, S. S. Fatahala, Acta Pharm. 2005, 55, 237-249.

41. W. M. Hussein, S. S. Fatahala, Z. M. Mohamed, R. P. McGeary, G. Schenk, D. L. Ollis, M. S. Mohamed, Chem. Biol. Drug Des. 2012, 80, 500-515. DOI:10.1111/j.1747-0285.2012.01440.x

42. M. S. Mohamed, R. Kamel, S. S. Fathallah, Arch. Pharm. (Weinheim) 2011, 344, 830-839.

DOI:10.1002/ardp.201100056

43. M. S. Mohamed, S. Ali, D. H. A. Abdelaziz, S. S. Fathallah, Biomed. Res. Int. 2014, 2014, 1-13.

44. K. M. H. Hilmy, E. B. Pedersen. Liebigs Ann. Chem. 1989, 1989, 1145-1146.

45. C. A. Winter, E. A. Risley, G. W. Nuss, J. Pharmacol. Exp. Ther. 1963, 141, 369-376.

46. E. C. Taylor, R. O. Kan, W. W. Paudler, J. Am. Chem. Soc. 1961, 83, 4484-4485. DOI:10.1021/ja01482a056

47. E. C. Taylor, R. W. Hendess, J. Am. Chem. Soc. 1965, 87, 19952003. DOI:10.1021/ja01087a025

48. E. C. Taylor, B. Liu, J. Org. Chem. 2001, 66, 3726-3738. DOI:10.1021/jo0015801

49. E. Fanghänel, K. Gewald, K. Pütsch, K. Wagner, J. Prakt. Chem. 1969, 311, 388-394.

DOI:10.1002/prac. 19693110307

50. T. D. Duffy, D. G. Wibberley, J. Chem. Soc., Perkin I 1974, 1921-1929. DOI:10.1039/p19740001921 
51. Z. Puterová, A. Krutošíková, D. Végh, Arkivoc 2010, (i), 209_ 246.

52. Y. Ding, H. An, Z. Hong, J.-L. Girardet, Bioorg. Med. Chem. Lett. 2005, 15, 725-727. DOI:10.1016/j.bmcl.2004.11.019

53. T.-C. Chien, E. A. Meade, J. M. Hinkley, L. B. Townsend, Org. Lett. 2004, 6, 2857-2859. DOI:10.1021/ol049207d

54. M. Qian, R. Glaser, J. Am. Chem. Soc. 2004, 126, 2274-2275. DOI:10.1021/ja0389523

55. J. U. Jeong, X. Chen, A. Rahman, D. S. Yamashita, J. I. Luengo, Org. Lett. 2004, 6, 1013-1016. DOI:10.1021/ol049921v

56. R. W. Fischer, M. Misun, Org. Process Res. Dev. 2001, 5, 581586. DOI:10.1021/op010041v

57. C. G. Dave, N. D. Desai, J. Heterocycl. Chem. 1999, 36, 729733. DOI:10.1002/jhet.5570360325

58. A. O. Abdelhamid, A. M. Negm, I. M. Abbas, J. Prakt. Chemie 1989, 331, 31-36. DOI:10.1002/prac.19893310106

59. K. M. H. Hilmy, Arch. Pharm. (Weinheim) 2004, 337, 15-19. DOI:10.1002/ardp.200300773

60. M. S. Mohamed, S. S. Fathallah, Mini. Rev. Org. Chem. 2014, 6, 477-507.

61. D. W. Goheen, W. R. Vaughan, Org. Synth. 1959, 39, 37-39. DOI:10.15227/orgsyn.039.0037

62. McNelis, E. kon and E. J. Chem. Soc., Chem. Comm. 1973, 562-563.

63. J. Oh, C. Ziani-Cherif, J.-R. Choi, J. K. Cha, Org. Synth. 2002, 78, 212-219. DOI:10.15227/orgsyn.078.0212

64. P. M. Traxler, P. Furet, H. Mett, E. Buchdunger, T. Meyer, N. Lydon, J. Med. Chem. 1996, 39, 2285-2292.

DOI:10.1021/jm960118j

65. M. M. Ghorab, F. A. Ragab, H. I. Heiba, H. A. Youssef, M. G. El-Gazzar, Bioorg. Med. Chem. Lett. 2010, 20, 6316-6320. DOI:10.1016/j.bmcl.2010.08.005

66. C. G. Dave, R. D. Shah, Molecules 2002, 7, 554-565. DOI:10.3390/70700554

67. C. Teixeira, F. Barbault, J. Rebehmed, K. Liu, L. Xie, H. Lu, S. Jiang, B. Fan, F. Maurel, Bioorg. Med. Chem. 2008, 16, 30393048. DOI:10.1016/j.bmc.2007.12.034

68. M. Martín-Martínez, A. Marty, M. Jourdan, C. Escrieut, E. Archer, R. González-Muñiz, M. T. García-López, B. Maigret, R. Herranz, D. Fourmy, M. Marti, J. Med. Chem. 2005, 48, 4842-4850. DOI:10.1021/jm0501127
69. G. Cristalli, S. Costanzi, C. Lambertucci, S. Taffi, S. Vittori, R. Volpini, Farmaco 2003, 58, 193-204.

DOI:10.1016/S0014-827X(03)00019-3

70. Y.-D. Gao, D. Feng, R. P. Sheridan, G. Scapin, S. B. Patel, J. K. Wu, X. Zhang, R. Sinha-Roy, N. A. Thornberry, A. E. Weber, T. Biftu, Bioorg. Med. Chem. Lett. 2007, 17, 3877-3879.

DOI:10.1016/j.bmcl.2007.04.106

71. Molecular Operating Environment (MOE), 2013.08; Chemical Computing Group Inc.: 1010 Sherbooke St. West, Suite \#910, Montreal, QC, Canada, H3A 2R7, 2014. 2014.

72. M. Rarey, B. Kramer, T. Lengauer, G. Klebe, J. Mol. Biol. 1996, 261, 470-489. DOI:10.1006/jmbi.1996.0477

73. D. M. Lorber, B. K. Shoichet, Protein Sci. 1998, 7, 938-950. DOI:10.1002/pro.5560070411

74. A. Zarghi, S. Arfaei, Iran. J. Pharm. Res. 2011, 10, 655-683.

75. J. DeRuiter, In Principles of Drug Action; 2002; pp 1-26.

76. D. Choi, Y. L. Piao, Y. Wu, H. Cho, Bioorg. Med. Chem. 2013, 21, 4477-4484. DOI:10.1016/j.bmc.2013.05.049

77. T. H. Page, J. J. O. Turner, A. C. Brown, E. M. Timms, J. J. Inglis, F. M. Brennan, B. M. J. Foxwell, K. P. Ray, M. Feldmann, J. Immunol. 2010, 185, 3694-3701.

DOI:10.4049/jimmunol.1000906

78. R. Danesi, G. Pasqualetti, E. Giovannetti, F. Crea, G. Altavilla, M. Del Tacca, R. Rosell, Adv. Drug Deliv. Rev. 2009, 61, 408-417. DOI:10.1016/j.addr.2009.03.001

79. P. P. N. Rao, S. N. Kabir, T. Mohamed, Pharmaceuticals 2010, 3, 1530-1549. DOI:10.3390/ph3051530

80. S. Ushiyama, T. Yamada, Y. Murakami, S. I. Kumakura, S. I. Inoue, K. Suzuki, A. Nakao, A. Kawara, T. Kimura, Eur. J. Pharmacol. 2008, 578, 76-86.

DOI:10.1016/j.ejphar.2007.08.034

81. W. O. Foye, In Williams \& Wilkins; 2008.

82. M. R. Shaaban, T. S. Saleh, A. S. Mayhoub, A. Mansour, A. M. Farag, Bioorg. Med. Chem. 2008, 16, 6344-6352.

DOI:10.1016/j.bmc.2008.05.011

83. H. N. Hafez, O. K. Al-duaij, A. B. A. El-gazzar, International J. Org. Chem. 2013, 3, 110-118.

DOI:10.4236/ijoc.2013.32012

84. C. Limban, A. Missir, K. M. S. Fahelelbom, M. M. Al-Tabakha, M. T. Caproiu, B. Sadek, Drug Des. Devel. Ther. 2013, 7, 883-892.

\section{Povzetek}

Sintetizirali smo serijo pripojenih pirolov in jih in vivo testirali za njihovo aktivnost proti vnetjem. Med 14 preiskovanimi derivati smo ugotovili, da 5 derivatov (1b-e,g and $\mathbf{5 b}$ ) kaže obetavno aktivnost proti vnetjem. Njihova aktivnost je primerljiva $\mathrm{z}$ aktivnostjo referenčnih proti-vnetnih zdravil (indometacin in ibuprofen). Da bi interpretirali rezultate bioloških študij preiskovanih spojin, smo izvedli tudi študije molekulskega sidranja. Rezultati so bili komplementarni tistim, dobljenim z biološkimi testiranji; poleg tega so potrdili biološke učinke preiskovanih spojin. 